\title{
Hubble Space Telescope Imaging of the Ultra-compact High Velocity Cloud AGC 226067: A Stripped Remnant in the Virgo Cluster
}

\author{
D. J. Sand ${ }^{1}$, A. C. Seth ${ }^{2}$, D. Crnojevic ${ }^{1}$, K. Spekkens ${ }^{3}$, J. Strader ${ }^{4}$, E. A. K. Adams ${ }^{5}$, N. Caldwell ${ }^{6}$, P. Guhathakurta ${ }^{7}$ J. Kenney $^{8}$, \\ S. Randall ${ }^{6}$, J. D. Simon ${ }^{9}$, E. Toloba ${ }^{10}$, and B. Willman ${ }^{11,12}$ \\ ${ }^{1}$ Texas Tech University, Physics \& Astronomy Department, Box 41051, Lubbock, TX 79409-1051, USA; david.sand@ttu.edu \\ ${ }^{2}$ Department of Physics and Astronomy, University of Utah, Salt Lake City, UT 84112, USA \\ ${ }^{3}$ Royal Military College of Canada, Department of Physics, P.O. Box 17000, Station Forces, Kingston, Ontario, K7K 7B4, Canada \\ ${ }^{4}$ Center for Data Intensive and Time Domain Astronomy, Department of Physics and Astronomy, Michigan State University, \\ 567 Wilson Road, East Lansing, MI 48824, USA \\ ${ }^{5}$ ASTRON, Netherlands Institute for Radio Astronomy, Postbus 2, 7900 AA Dwingeloo, The Netherlands \\ ${ }^{6}$ Harvard-Smithsonian Center for Astrophysics, Cambridge, MA 02138, USA \\ ${ }^{7}$ UCO/Lick Observatory, University of California, Santa Cruz, 1156 High Street, Santa Cruz, CA 95064, USA \\ ${ }^{8}$ Yale University Astronomy Department, P.O. Box 208101, New Haven, CT 06520-8101, USA \\ ${ }^{9}$ Observatories of the Carnegie Institution for Science, 813 Santa Barbara Street, Pasadena, CA 91101, USA \\ ${ }_{10}$ Department of Physics, University of the Pacific, 3601 Pacific Avenue, Stockton, CA 95211, USA \\ ${ }^{11}$ Steward Observatory, University of Arizona, 933 North Cherry Avenue, Tucson, AZ 85721, USA \\ ${ }^{12}$ LSST, University of Arizona, 933 North Cherry Avenue, Tucson, AZ 85721, USA \\ Received 2017 February 20; revised 2017 May 16; accepted 2017 May 24; published 2017 July 13
}

\begin{abstract}
We analyze the optical counterpart to the ultra-compact high velocity cloud AGC 226067, utilizing imaging taken with the Advanced Camera for Surveys (ACS) on the Hubble Space Telescope. The color-magnitude diagram of the main body of AGC 226067 reveals an exclusively young stellar population, with an age of $\sim 7-50 \mathrm{Myr}$, and is consistent with a metallicity of $[\mathrm{Fe} / \mathrm{H}] \sim-0.3$ as previous work has measured via $\mathrm{H}$ II region spectroscopy. Additionally, the color-magnitude diagram is consistent with a distance of $D \approx 17 \mathrm{Mpc}$, suggesting an association with the Virgo cluster. A secondary stellar system located $\sim 1 ! 6(\sim 8 \mathrm{kpc})$ away in projection has a similar stellar population. The lack of an old red giant branch $(\gtrsim 5 \mathrm{Gyr})$ is contrasted with a serendipitously discovered Virgo dwarf in the ACS field of view (Dw J122147+132853), and the total diffuse light from AGC 226067 is consistent with the luminosity function of the resolved $\sim 7-50 \mathrm{Myr}$ stellar population. The main body of AGC 226067 has a $M_{V}=-11.3 \pm 0.3$, or $M_{\text {stars }}=5.4 \pm 1.3 \times 10^{4} M_{\odot}$ given the stellar population. We searched $20 \mathrm{deg}^{2}$ of imaging data adjacent to AGC 226067 in the Virgo Cluster, and found two similar stellar systems dominated by a blue stellar population, far from any massive galaxy counterpart-if this population has star-formation properties that are similar to those of AGC 226067, it implies $\sim 0.1 M_{\odot} \mathrm{yr}^{-1}$ in Virgo intracluster star formation. Given its unusual stellar population, AGC 226067 is likely a stripped remnant and is plausibly the result of compressed gas from the ram pressure stripped M86 subgroup ( $\sim 350 \mathrm{kpc}$ away in projection) as it falls into the Virgo Cluster.
\end{abstract}

Key words: galaxies: dwarf

\section{Introduction}

Searching for faint, isolated dwarf galaxies in large area $\mathrm{H} \mathrm{I}$ surveys has a long history (see Giovanelli \& Haynes 2016 for a review). Most recently, so-called ultra-compact high velocity clouds (UCHVCs) of neutral hydrogen have been identified by the Galactic Arecibo $L$-Band Feed Array H I (GALFA-H I) and Arecibo Legacy Fast Arecibo $L$-Band Feed Array (ALFALFA) surveys (Saul et al. 2012; Adams et al. 2013, respectively) as potential sites of gas-bearing dark matter halos. Because the spatial distribution and physical properties of these UCHVCs are consistent with some being faint dwarf galaxies in the Local Volume (e.g., Giovanelli et al. 2010; Adams et al. 2013), several searches have been undertaken to characterize their optical properties (Bellazzini et al. 2015a; Sand et al. 2015; Tollerud et al. 2015; Janesh et al. 2015). These searches have yielded only a handful of plausible dwarf galaxy counterparts to the UCHVCs, while follow-up H I observations of others suggest that some may be "Dark" systems (e.g., Adams et al. 2016). Most, however, are likely Galactic H I clouds.

The subject of this paper is Hubble Space Telescope (HST) imaging of AGC $226067\left(v_{\odot}=-140 \mathrm{~km} \mathrm{~s}^{-1}\right.$; also referred to as SECCO1), a UCHVC with an intriguing optical counterpart.
First identified by Bellazzini et al. (2015a), it was confirmed spectroscopically to be associated with the coincident $\mathrm{H} \mathrm{I}$ cloud (Bellazzini et al. 2015b; Sand et al. 2015). Follow-up highresolution $\mathrm{H}$ I observations with the Very Large Array showed that AGC 226067 broke up into two distinct H I clouds (Adams et al. 2015) - the primary H I source is coincident with the optical/UV counterpart, while the secondary H I component also has an optical/UV counterpart offset by 0'5. Data from the Multi Unit Spectroscopic Explorer (MUSE) at the VLT revealed 38 separate $\mathrm{H}$ II regions scattered throughout the main and secondary stellar body of AGC 226067, with a nearly uniform metallicity of $\langle 12+\log (\mathrm{O} / \mathrm{H})\rangle=8.37$ (for the main body), more metal-rich than expected if AGC 226067 was a gas-rich dwarf galaxy (Beccari et al. 2017a). AGC 226067 is projected onto the "Low Velocity Cloud" region of the Virgo Cluster (at $D \approx 17 \mathrm{Mpc}$, which we use throughout this work; Boselli et al. 2014), which has a velocity distribution centered at $v_{\mathrm{LSR}} \sim 0 \mathrm{~km} \mathrm{~s}^{-1}$ with a range of $\pm 400 \mathrm{~km} \mathrm{~s}^{-1}$. For simplicity, we refer to the entire system (consisting of the main and secondary stellar bodies, along with the two H I clouds) as AGC 226067 throughout this work, and will refer to particular regions of the system (e.g., its "main" and 


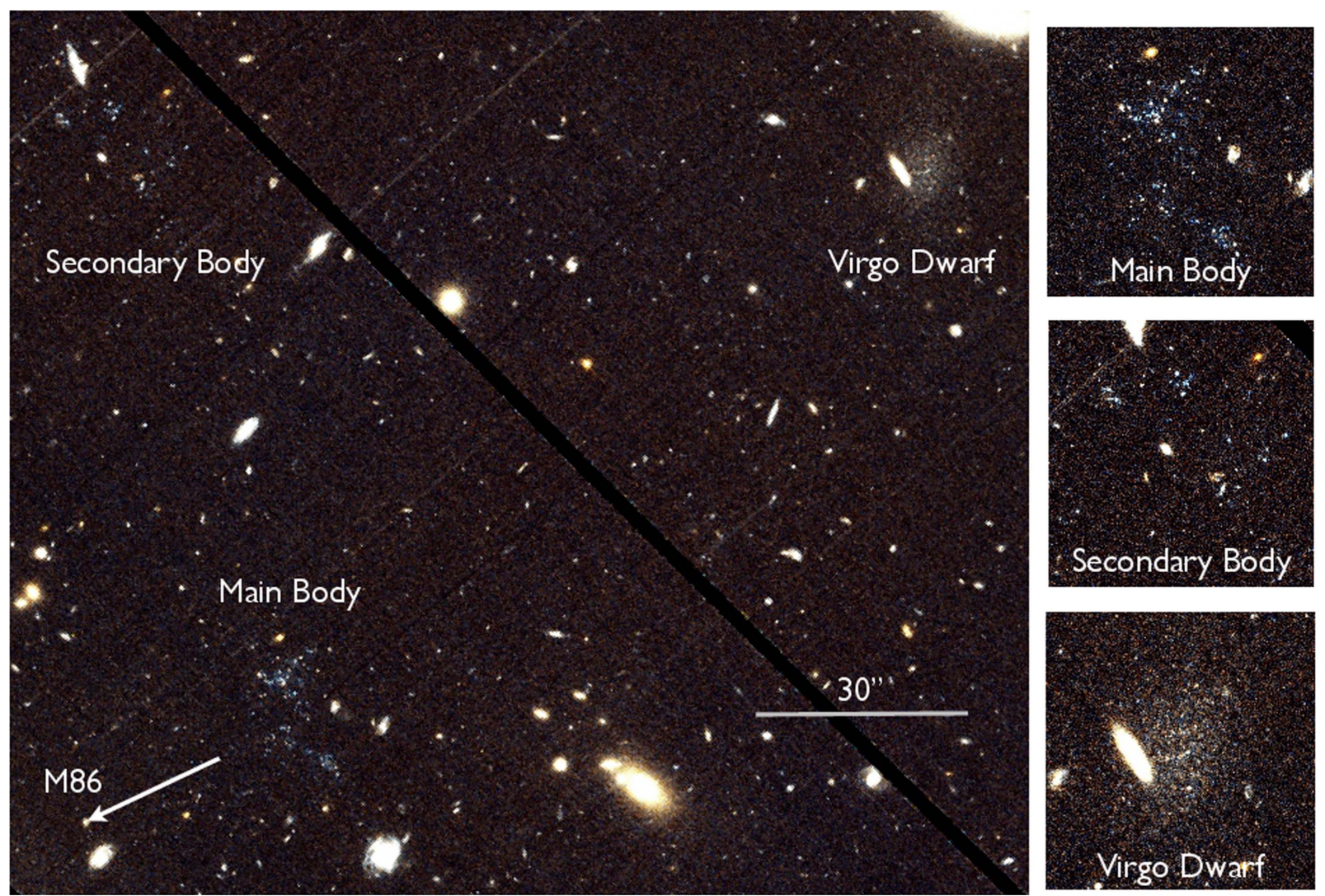

Figure 1. Left: a colorized cutout of the F606W and F814W HST/ACS image of AGC 226067 and its surroundings; several notable regions are highlighted. North is up and east is to the left. The M86 subgroup is in the southwest direction. Right column: from top to bottom, we show zoom-ins of the main body of AGC 226067, its secondary body and a newly discovered Virgo dwarf galaxy, Dw J122147+132853.

"secondary" body) when getting into specifics. In addition to a presentation of the HST data (Section 2) and an analysis of the stellar population of AGC 226067 (Section 3), we perform an initial optical/UV archival search for similar stellar systems (Section 4) in deep Virgo cluster data. We discuss possible origins for AGC 226067 in Section 5, and then conclude in Section 6.

\section{Hubble Space Telescope Observations and Data Reduction}

HST observations of AGC 226067 (GO 13735; PI Sand) were taken with ACS (Ford et al. 1998) in the F606W (2196 s) and F814W (2336 s) filters on 2015 April 26, as well as the Wide Field Camera 3 (WFC3) in the F275W filter (2470 s) on 2015 July 7. The WFC3 F275W data contained only a handful of low $\mathrm{S} / \mathrm{N}$ sources, and we will not consider it further. For the F606W and F814W data, multiple exposures were taken in each filter to remove cosmic rays, but we did not dither to fill in the chip gaps because this was not necessary to fully image AGC 226067.

Point-spread function photometry was performed using the software package DOLPHOT (Dolphin 2000) on the individual charge transfer efficiency corrected ACS images (the FLC files), with input parameters similar to Williams et al. (2014). Sources with $\left(\operatorname{sharp}_{\mathrm{F} 606 \mathrm{~W}}+\operatorname{sharp}_{\mathrm{F} 814 \mathrm{~W}}\right)^{2}>0.1$ and $\left(\operatorname{crowd}_{\mathrm{F} 606 \mathrm{~W}}+\right.$ crowd $\left._{\mathrm{F} 814 \mathrm{~W}}\right)^{2}>1.0$ were culled, and only those remaining sources with a signal-to-noise ratio greater than 4 in both the F606W and F814W bands were kept for further analysis. Artificial star tests were also run to quantify our photometric uncertainties and completeness; the 50\% completeness limits are shown in Figure 2. The final photometric catalog was corrected for Galactic extinction (Schlafly \& Finkbeiner 2011) corresponding to the position of the main body of AGC 226067, with an adopted value of $E(B-V)=0.057$. All magnitudes presented in this work have this correction applied. All results are presented in the VEGAMAG system.

To create mosaics of the F606W and F814W ACS images, we used Drizzlepac v2.0 and the ASTRODRIZZLE routine. In Figure 1, we present a false color red giant branch (RGB) image of AGC 226067 and its surroundings (using the average of the F606W and F814W images as our "green" image), with zoomed in cutouts highlighting several points of interest. The main stellar body of AGC 226067 consists primarily of blue stars in several distinct clumps. A second clump of blue stars $\sim 1.6$ arcmin to the northwest of the main body of AGC 226067 is seen, as has been noted in previous work (Adams et al. 2015; Sand et al. 2015; Beccari et al. 2016, 2017a). In contrast, an uncataloged Virgo cluster dwarf galaxy serendipitously in our ACS field of view (which we dub Dw J122147+132853) consists almost entirely of RGB stars, which we discuss below.

\section{Properties of AGC 226067}

\subsection{Stellar Population}

The color-magnitude diagram (CMD) of AGC 226067 reveals a complex and exclusively young stellar population. In the top panel of Figure 2, we show the CMD of AGC 226067 centered on its main body, along with several 

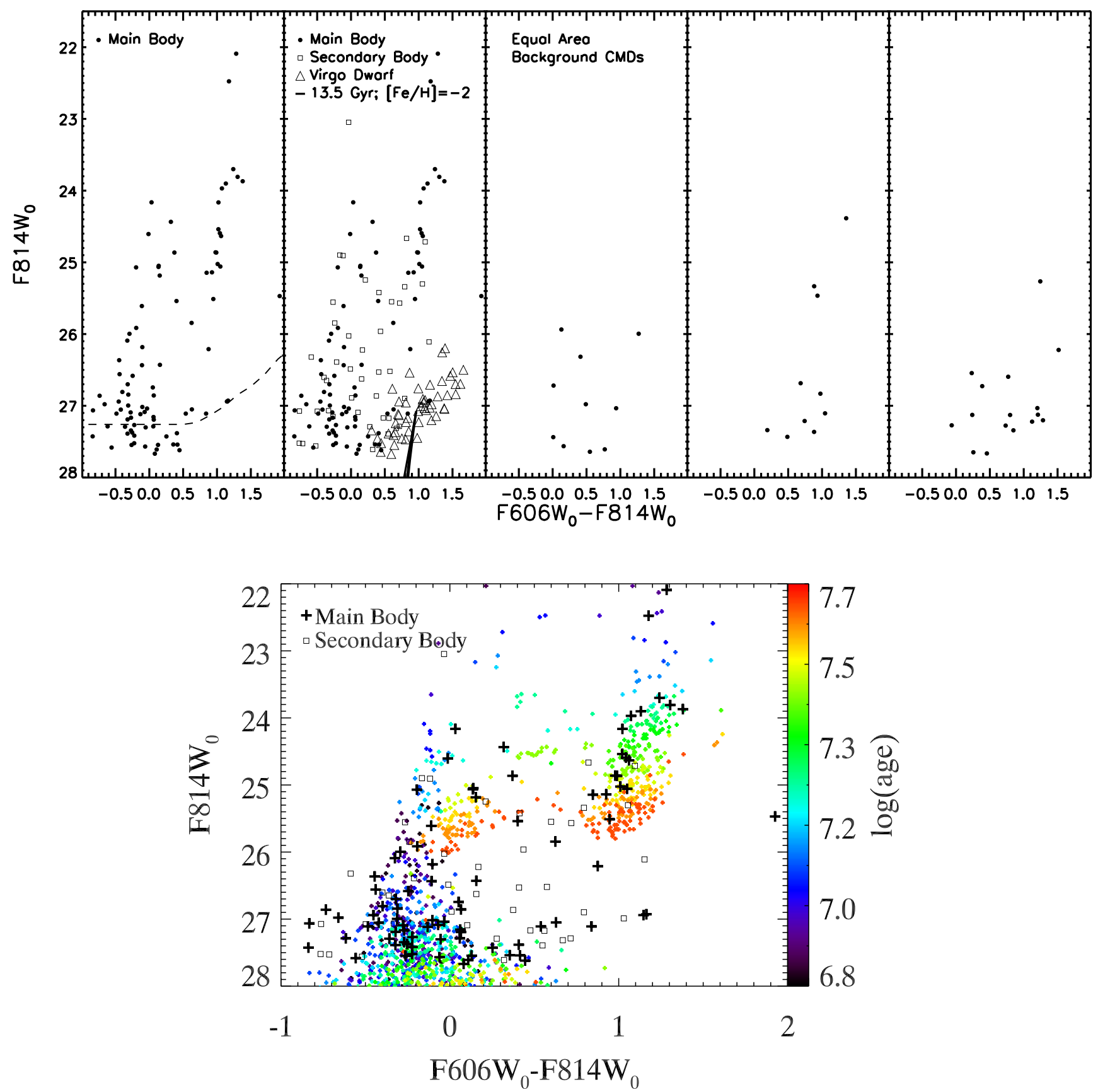

Figure 2. Top: the left panel shows the CMD of the main body of AGC 226067, followed by the CMDs of the secondary body and Dw J122147+132853. While the secondary body has a CMD similar to that of the main body, Dw J122147+132853 has a clear RGB population that is not apparent in AGC 226067. The three adjacent panels show equal area background CMDs. The CMD of AGC 226067 shows an overdensity of blue stars and an RHeB sequence. The dashed line in the far left panel shows the 50\% completeness limit. In Table 1, we list the position and area over which each of the non-background CMDs is drawn from. Bottom: the CMD of the main and secondary body, along with a simulated stellar population with a constant star-formation history between $\sim 7-50 \mathrm{Myr}$ and a $[\mathrm{Fe} / \mathrm{H}] \sim-0.3$, which matches the extent of the observed $\mathrm{RHeB}$ sequence.

background CMDs drawn from equal area outlying regions. AGC 226067's main body has a population of faint, blue stars $\left(\mathrm{F}_{606 \mathrm{~W}_{0}}-\mathrm{F} 814 \mathrm{~W}_{0}<0.0\right.$ and $\left.\mathrm{F} 814 \mathrm{~W}_{0} \gtrsim 26 \mathrm{mag}\right)$ that are likely young main-sequence stars, along with a sequence of stars

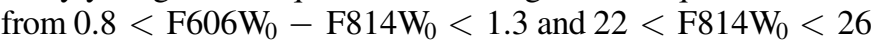
mag, which are consistent with being red helium burning (RHeB) stars. There are only a handful of possible old, RGB stars (at $\mathrm{F}_{606 \mathrm{~W}_{0}}-\mathrm{F} 814 \mathrm{~W}_{0}>0.4$ and $\mathrm{F} 814 \mathrm{~W}_{0} \gtrsim 27$ ). The morphology of AGC 226067 is broken up into several clumps with an approximate spatial extent of $\sim 20^{\prime \prime}(\sim 1.6 \mathrm{kpc}$; Figure 1$)$.
Also shown in Figure 2, we overplot the CMDs of the secondary body (to be discussed in Section 3.2) and the new Virgo dwarf Dw J122147+132853, along with a $13.5 \mathrm{Gyr}$, $[\mathrm{Fe} / \mathrm{H}]=-2$ isochrone (Bressan et al. 2012). There is no apparent overdensity of RGB stars associated with AGC 226067, signaling that any old stellar population must be small. This absence of RGB stars becomes more striking when compared with the CMD of Dw J122147+132853, which shows a large population of such stars (compatible with our adopted $D=17 \mathrm{Mpc}$ ). A simple largeaperture analysis (see Sand et al. 2014, with masking of the nearby 
background spiral galaxy) indicates that Dw J122147+132853 has an absolute magnitude of $M_{\mathrm{F} 606 \mathrm{~W}}=-11.3 \pm 0.2$ and $M_{\mathrm{F} 814 \mathrm{~W}}=$ $-11.7 \pm 0.2$; this is $M_{V}=-11.4 \pm 0.2$ using the transformations of Sirianni et al. (2005).

The brightness of RHeB stars in optical CMDs is directly dependent on the age of the star (McQuinn et al. 2011), which we will use to constrain the stellar population in AGC 226067. In the bottom panel of Figure 2, we show the results of simulating a constant star-formation history $1 \times 10^{6} \mathrm{M}_{\odot}$ stellar population with ages between $\sim 7-50 \mathrm{Myr}$ and a $[\mathrm{Fe} / \mathrm{H}]=-0.3$ (corresponding to the gas-phase metallicity found by Beccari et al. 2017a, assuming the solar abundance of Grevesse et al. 2007) at a distance of $17 \mathrm{Mpc}$ (we assumed a Kroupa initial mass function; Kroupa 2001). We have convolved the simulated data set with our measured photometric uncertainties. When compared with the CMD of AGC 226067's main body, the dimmest RHeB stars correspond to an age of $\sim 50 \mathrm{Myr}$, while the two bright stars at the top of the sequence $\left(\mathrm{F} 814 \mathrm{~W}_{0} \approx 22-22.5\right)$ correspond to $\mathrm{RHeB}$ stars of $\sim 7-8 \mathrm{Myr}$ - we infer an approximate stellar population with an age range of $\sim 7-50 \mathrm{Myr}$ for AGC 226067. Star formation could have continued to the present day at a similar rate, but our CMDs cannot constrain this due to low number statistics. The simulated stellar population is not a perfect match to the colors of the observed CMD, but this is likely due to known limitations of stellar evolution models at these young ages (i.e., uncertainties associated with internal mixing, stellar rotation, and mass loss; Dohm-Palmer \& Skillman 2002; McQuinn et al. 2011). The age of the youngest population of stars is consistent with the observation that AGC 226067 has $\mathrm{H}$ II regions.

We estimate the stellar mass of the main body of AGC 226067 by directly comparing the relative number of RHeB stars to that of our simulated $1 \times 10^{6} M_{\odot}$ stellar population, subtracting a background derived from outlying regions of the ACS field of view. We find $M_{\text {stars }}=5.4 \pm 1.3 \times 10^{4} M_{\odot}$ (where the error bar includes the effects of Poisson statistics and background subtraction, but not variations in stellar models or star-formation histories), which is consistent with our finding from direct aperture photometry on the main body (Section 3.4). Note that the photometric completeness at the faint end of the RHeB sequence is $>95 \%$, and so cannot effect our results. This and other properties of AGC 226067 are shown in Table 1.

We can use this stellar mass to obtain an estimate of the starformation rate (SFR) of the main body; assuming uniform star formation over the past $50 \mathrm{Myr}$, we obtain an SFR of $1.1 \times 10^{-3} M_{\odot} \mathrm{yr}^{-1}$. This is in general agreement with revised values of the SFR based off the MUSE spectra (Beccari et al. 2017b).

We can also look at the nature of star formation in this object by examining where it falls on a Kennicutt-Schmidt relation of SFR density $\left(\Sigma_{\mathrm{SFR}}\right)$ versus gas density. Assuming our SFR estimate for the main body and a radius of $1.1 \mathrm{kpc}$ (Table 1), we get a $\Sigma_{\mathrm{SFR}}=2.9 \times 10^{-4} M_{\odot} \mathrm{yr}^{-1} \mathrm{kpc}^{-2}$. The main body has an estimated $\mathrm{H}$ I mass of $1.5 \times 10^{7} M_{\odot}$ in a deconvolved area of $3.08 \times 10^{7} \mathrm{pc}^{2}$ (Adams et al. 2015), giving a gas surface density of $0.49 M_{\odot} \mathrm{pc}^{-2}$. These values suggest the star formation in AGC 226067 is occurring at the low end of gas surface densities in comparison to nearby galaxy disks (Bigiel et al. 2008) and is slightly more efficient than that typically seen in the outer regions of nearby spirals and dwarfs (Bigiel et al. 2010).
Table 1

Properties of AGC 226067 and Other Stellar Systems

\begin{tabular}{|c|c|}
\hline Parameter & Value \\
\hline \multicolumn{2}{|c|}{ AGC 226067 Main Body } \\
\hline R.A. (h:m:s) & $12: 21: 54.04$ \\
\hline Decl. (d:m:s) & $+13: 27: 35.7$ \\
\hline Radial size (arcsec) & 13.7 \\
\hline Radial size (kpc) & 1.1 \\
\hline$M_{V}$ & $-11.3 \pm 0.3$ \\
\hline$M_{\text {stars }}\left(10^{4} M_{\odot}\right)$ & $5.4 \pm 1.3$ \\
\hline$M_{\mathrm{H} \mathrm{I}}\left(10^{7} M_{\odot}\right)^{\mathrm{a}}$ & 1.5 \\
\hline$M_{\mathrm{H} \mathrm{I}} / M_{\text {stars }}$ & $\approx 280$ \\
\hline \multicolumn{2}{|c|}{ AGC 226067 Secondary Body } \\
\hline R.A. (h:m:s) & $12: 21: 55.97$ \\
\hline Decl. (d:m:s) & $+13: 28: 53.6$ \\
\hline Radial size (arcsec) & 13.0 \\
\hline Radial size (kpc) & 1.1 \\
\hline$M_{\text {stars }}\left(10^{3} M_{\odot}\right)$ & $5.5 \pm 4.2$ \\
\hline \multicolumn{2}{|l|}{ Dw J122147+132853 } \\
\hline R.A. (h:m:s) & $12: 21: 47.87$ \\
\hline Decl. (d:m:s) & $+13: 28: 54.7$ \\
\hline Radial size (arcsec) & 10.0 \\
\hline Radial size (kpc) & 0.8 \\
\hline$M_{V}$ & $-11.4 \pm 0.2$ \\
\hline \multicolumn{2}{|c|}{ NGVS-1+2 J122624+142327 } \\
\hline R.A. (h:m:s) & $12: 21: 24$ \\
\hline Decl. (d:m:s) & $+14: 23: 27$ \\
\hline$g$ & $20.1 \pm 0.6$ \\
\hline$N U V$ & $19.5 \pm 0.3$ \\
\hline \multicolumn{2}{|c|}{ NGVS-1+3 J122631+151026 } \\
\hline R.A. (h:m:s) & $12: 26: 31$ \\
\hline Decl. (d:m:s) & $+15: 10: 26$ \\
\hline$g$ & $20.7 \pm 0.5$ \\
\hline$N U V$ & $20.8 \pm 0.7$ \\
\hline
\end{tabular}

Note. The coordinates and radial size for AGC 226067 and Dw J122147 +132853 are approximate, but they correspond to the CMD region used in Figure 2.

${ }^{\mathrm{a}}$ From Adams et al. (2015).

\subsection{The Secondary Body}

Several previous works have also pointed out a secondary clump of blue stars (Figure 1$)$, located $\sim 1$ ! $6(\sim 8 \mathrm{kpc})$ to the northeast of the main body of AGC 226067 (Adams et al. 2015; Sand et al. 2015; Beccari et al. 2016, 2017a). A CMD of this region is shown in the middle-left panel at the top of Figure 2. The stars in this secondary knot are consistent with the stellar population of the main body of AGC 226067. Although it is not sufficiently populated to confirm the presence of stars with age $<10 \mathrm{Myr}$, it is likely the case given the secondary clump has several H II regions (Beccari et al. 2017a). Again, there are few old RGB stars, in stark contrast to the nearby Virgo dwarf. We estimate the stellar mass in the secondary body identically to the technique employed on the main body and find $M_{\text {stars }}=5.5 \pm 4.2 \times 10^{3} M_{\odot}$ (see Table 1). 


\subsection{Other Possible Stellar Extensions of AGC 226067}

Two other regions around AGC 226067 have been discussed in the literature as possible physically associated extensions, and we examine our $H S T$ photometry at these positions in more detail here.

The first is an additional clump of $\mathrm{H} \mathrm{I}$ gas, discovered and dubbed AGC 229490 by Adams et al. (2015), which is physically separated from the main body of AGC 226067 , but has a consistent $\mathrm{H}$ I-derived velocity $\left(v_{\odot}=-123\right.$ and $-142 \mathrm{~km} \mathrm{~s}^{-1}$, respectively). We have constructed a CMD of AGC 229490 corresponding to the position of the gas cloud, and its rough dimensions (R.A.: 12:21:53.8, decl.: +13:29:08, with size $45^{\prime \prime} \times 45^{\prime \prime}$; Adams et al. 2015). The CMD is largely consistent with several equal area background CMDs, possibly with an overdensity of $\sim 5$ or fewer stars. Thus the secondary H I cloud, AGC 229490, is largely star-free.

More recently, a deep ground-based image of the AGC 226067 field suggested the presence of an additional blue stellar overdensity located $\sim 1$ arcmin to the northeast of the main body (Beccari et al. 2016). A CMD of the region again suggests that this field consists of primarily foreground stars, though there are a handful of stars that are consistent with a 7-50 Myr stellar population.

\subsection{Constraints on an Old Stellar Population}

The lack of RGB stars in the CMD of AGC 226067 is striking when compared with the serendipitous Virgo dwarf galaxy in our ACS field of view, which has an absolute magnitude of $M_{V} \approx-11.4$. We take two approaches for constraining an old stellar population in AGC 226067's main body.

First, we use the number of stars in the region of the CMD where we find RGB stars in Dw J122147+132853 to constrain the old stellar mass of AGC 226067. We defined a CMD box surrounding the stars within Dw J122147 +132853 (with $\mathrm{F} 606 \mathrm{~W}_{0}-\mathrm{F}_{814 \mathrm{~W}_{0}}$ between 0.30 and 1.66 and $\mathrm{F} 814 \mathrm{~W}_{0}$ between 26.20 and 27.67); after background subtraction, we find $43 \pm 7$ in Dw J122147+132853. However, for the main body of AGC 226067, we find only $4 \pm 4$ stars. Using the absolute magnitude of Dw J122147+132853 $\left(M_{V}=-11.4\right)$, this translates to a limiting absolute magnitude for the old population of AGC 226067 of $M_{V}>-8.8 \mathrm{mag}$, or $M_{\text {stars }}<6 \times 10^{5} M_{\odot}$ (assuming a $M / L_{V}=2 M_{\odot} / L_{\odot}$ for an old stellar population; Strader et al. 2011; Baumgardt 2017). This limit on the old stellar population in AGC 226067 hinges on it being at the same distance as Dw J122147+132853; if it were at a greater relative distance, the old stellar population's limits would be weaker. Similarly, if any putative old stellar population in AGC 226067 were significantly more metal-rich than in Dw J122147+132853 its RGB stars would be pushed to relatively redder colors, outside of our CMD selection box. This relative difference in metallicity would again cause us to underestimate the old stellar population in AGC 226067. Finally, if a population of older stars is associated with AGC 226067, but spread out over a larger area than the young stellar population, then our limits on an old stellar population would be proportionately brighter (assuming a constant stellar surface density).

We measure the integrated magnitude for the main body within an 11" radius around AGC 226067 (after masking background galaxies) of $M_{\mathrm{F} 606 \mathrm{~W}, 0}=-11.3 \pm 0.3$ and $M_{\mathrm{F} 814 \mathrm{~W}, 0}=-11.2 \pm 0.3$, translating to $M_{V}=-11.3 \pm 0.3$. For the age range of 7-50 Myr, the Bruzual \& Charlot (2003) models suggest $M / L_{V}=0.013-0.06 M_{\odot} / L_{\odot}$; this suggests a stellar mass of $M_{\text {stars }}=4-17 \times 10^{4} M_{\odot}$, consistent with our measurement of $M_{\text {stars }}=5.4 \pm 1.3 \times 10^{4} M_{\odot}$ in Section 3.1. This measured absolute magnitude is fully consistent with previous ground-based data (e.g., Adams et al. 2015), but is $\sim 2 \sigma$ brighter than Beccari et al. (2017a).

We also subtracted the point sources in our DOLPHOT catalog from the F606W and F814W ACS mosaic images, and have analyzed the remaining diffuse light to constrain an old stellar population. Using this image and the same $11^{\prime \prime}$ annulus, we measure a diffuse, star-subtracted magnitude of $M_{\mathrm{F} 606 \mathrm{~W}, 0}=-9.8 \pm 0.4$ and $M_{\mathrm{F} 814 \mathrm{~W}, 0}=-10.0 \pm 0.5$, which corresponds to $M_{V}=-9.8 \pm 0.5 \mathrm{mag}$. The errors on these measurements include the range of values obtained using direct galaxy removal and background annulus subtraction.

We can compare the amount of diffuse light to that expected from the faint unresolved stars of the $\sim 7-50 \mathrm{Myr}$ stellar population revealed in the CMD, but below the detection limit of the ACS data. To estimate this, we first calculated the total magnitude of resolved stars consistent with this population in the main body of AGC 226067 and used it as an anchor for integrating the luminosity function below our ACS detection limit, assuming a 7-50 Myr stellar population with $[\mathrm{Fe} / \mathrm{H}]=-0.3$ and the associated Padova luminosity function (Bressan et al. 2012) and a Chabrier initial mass function. Depending on the choice of age of the stellar population, and the filter, our resolved stellar sources should make up $~ 45 \%-$ $70 \%$ of the total luminosity of AGC 226067, implying a diffuse stellar component from the young stellar population with $M_{V}=-10.4 \pm 0.2 \mathrm{mag}$ (where the error bars encapsulate the allowed range when varying the age of the stellar population). Thus, the observed diffuse component of AGC 226067 (directly measured as $M_{V}=-9.8 \pm 0.5 \mathrm{mag}$ ) is fully consistent with a stellar population belonging solely to the apparent young stellar population, with no need to invoke any associated older population.

Given the apparent lack of an old stellar population, AGC 226067 cannot be considered a typical dwarf irregularlike galaxy because these systems all have an old RGB population at $H S T$ depths (e.g., McQuinn et al. 2014). This strongly suggests that AGC 226067 is analogous to so-called "tidal dwarfs" (see Duc 2012 for a recent review) even if its origin is not from direct tidal interactions (see Section 5 for a discussion). Unlike previous examples of tidal dwarfs, AGC 226067 is extremely isolated, $\sim 350 \mathrm{kpc}$ projected distance from the nearest large galaxies.

\section{Similar Stellar Systems in the Virgo Cluster}

The optical appearance of AGC 226067 in three-color RGB images, combined with its strong detection in GALEX imaging, is striking (see Sand et al. 2015). Motivated to understand the uniqueness of AGC 226067 we undertook a search of a 20 $\mathrm{deg}^{2}$ region in the Next Generation Virgo cluster Survey (NGVS) footprint to identify similar systems.

The NGVS is a $\sim 100 \mathrm{deg}^{2}$ optical imaging survey conducted with the MegaCam imager on the Canada-France-Hawaii Telescope (CFHT); details are described in Ferrarese et al. (2012). The typical point-source depth was $g \approx 25.9$ mag $(10 \sigma)$. We downloaded fields from the CFHT archive, utilizing 

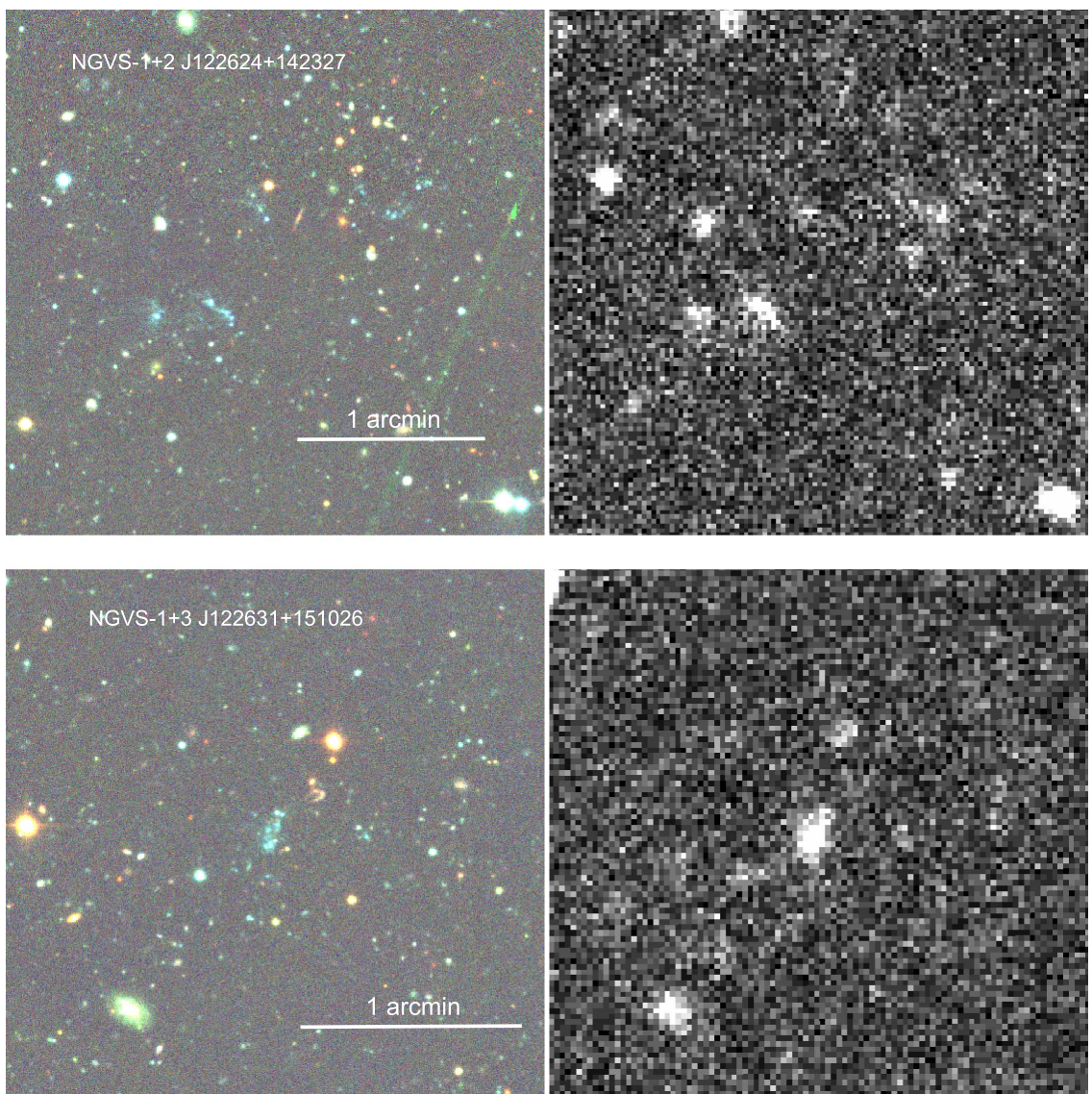

Figure 3. Color RGB optical (left) and GALEX NUV (right) images of the two stellar systems that look similar to AGC 226067 in a preliminary search of 20 deg ${ }^{2}$ of NGVS archival data. Both candidates project far from massive Virgo cluster galaxies and appear dominated by young stellar populations without a diffuse galaxy-like component. North is up and east is to the left.

the MegaPipe data products (Gwyn 2008), in the rectangular region demarcated by fields $[1,1]$ to $[-2,-3]$, using the nomenclature presented in Figure 4 of Ferrarese et al. (2012). Color RGB images were made with the $u$-, $g$-, and $i$-band data, and searched by two of us (D.J.S. and D.C.). We purposely avoided areas directly adjacent to prominent Virgo cluster galaxies, which often have blue, knotty extensions indicative of extended star formation (e.g., Thilker et al. 2007) and have a similar appearance as our more isolated examples. GALEX (Martin et al. 2005) imaging was used to support our candidate detections.

Two candidates visually similar to AGC 226067 were identified, and are shown in Figure 3. Each is characterized by an overdensity of compact blue sources with strong GALEX UV emission. The objects also lack a diffuse reddish galaxy component, exhibited by dwarf galaxies throughout the NGVS imaging. Aperture photometry (Sand et al. 2014) is reported for each object in Table 1; the objects are of similar brightness as AGC 226067 in the $N U V, g$ bands to within the uncertainties (Sand et al. 2015). We searched for H I counterparts to our newly identified blue stellar systems using the $40 \%$ ALFALFA catalog (Haynes et al. 2011). No cataloged H I counterparts were found to either source within $\sim 10^{\prime}$, and a visual inspection of the ALFALFA data did not turn up any marginal detections. Assuming that these objects are similar to AGC 226067, with $D=17 \mathrm{Mpc}$ and velocity width $\sim 50 \mathrm{~km} \mathrm{~s}^{-1}$, we estimate an $\mathrm{H} \mathrm{I}$ limiting mass of $\sim 2 \times 10^{7} M_{\odot}$ for each. AGC 226067 itself has an $M_{\mathrm{H}} \approx 5 \times 10^{7} M_{\odot}$ in the ALFALFA catalog, so a similar H I cloud with a slightly higher distance and/or velocity width would not be detectable with the ALFALFA data. We also note that ALFALFA is blind to emission that spectrally overlaps with the Galactic H I layer at $-100 \lesssim v_{\text {lsr }}\left(\mathrm{km} \mathrm{s}^{-1}\right) \lesssim 100$ along the line of sight, a velocity range in which they could plausibly be located given that Virgo's Low Velocity cloud has $v_{\mathrm{lsr}} \approx 0 \mathrm{~km} \mathrm{~s}^{-1}$.

In a future contribution, we will present a full search of the NGVS data set for similar blue, diffuse objects as well as follow-up optical/H I observations. The environment of these diffuse sources will also be investigated, though we note that NGVS-1+2 J122624+142327 is $\sim 28^{\prime}(\sim 130 \mathrm{kpc})$ from NGC 4377, while NGVS-1+3 J122631+151026 is $\sim 10^{\prime}$ ( $\sim 50 \mathrm{kpc})$ from NGC 4419, suggesting their possible association. Assuming a uniform distribution and given the relative ratio of the entire NGVS and our current search, we can expect to find a sample of $\sim 10-15$ systems in total, including AGC 226067 and the newly identified objects. Taking our inferred SFR of the main body of AGC $226067(1.1 \times$ $10^{-3} M_{\odot} \mathrm{yr}^{-1}$ ) as representative of the population as a whole, it implies an intracluster SFR of $\sim 0.01 M_{\odot} \mathrm{yr}^{-1}$ in the Virgo cluster. The metallicity of the newly forming stars are comparable to the younger component of intracluster stars (Williams et al. 2007) and the inferred rate is consistent with the loose upper limit on the intracluster SFR found in cluster supernova searches (Graham et al. 2012). The detected population may be the tip of the iceberg, as we likely missed 


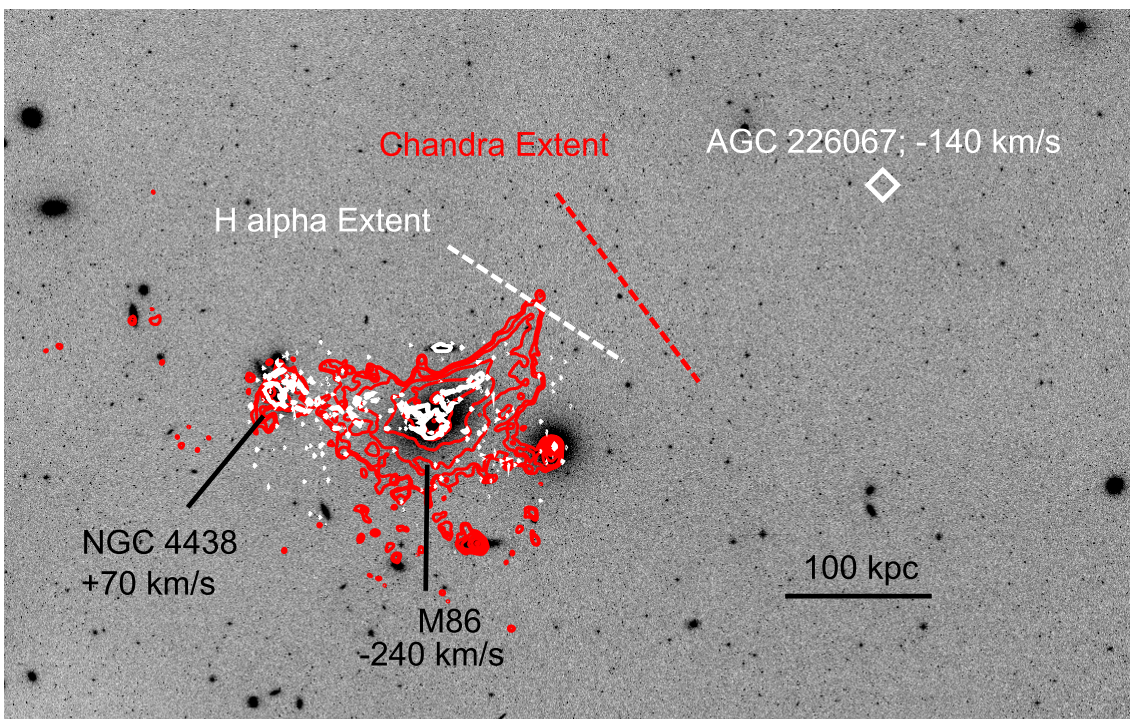

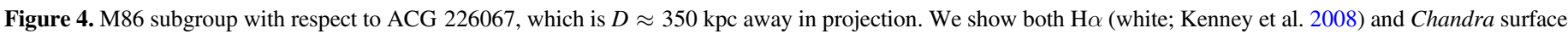

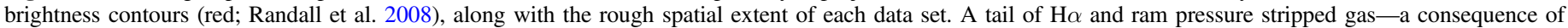
NGC 4438 and M86's complicated interaction with each other and the Virgo intracluster medium-is pointing roughly in the direction of AGC 226067.

less luminous examples, and older stellar populations are more difficult to find because their UV/blue flux fades. In the past, infalling gas-rich systems were likely more abundant than they are today, thus this process could contribute substantially to the observed population of intracluster stars.

\section{Discussion}

The properties of AGC 226067 and its immediate environs are intriguing. In this work, we have shown that the main body has an exclusively young stellar population, with age $\sim 7-50 \mathrm{Myr}$ and $[\mathrm{Fe} / \mathrm{H}] \approx-0.3$, with a $M_{V} \approx-11.3$ and $M_{\text {star }} \approx 5 \times 10^{4} M_{\odot}$. There is a secondary group of stars located $\sim 1.5(\sim 7.5 \mathrm{kpc})$ to the northeast, which is sparse but has a stellar population consistent with the main body of AGC 226067. The entire stellar system hosts a complex of $\mathrm{H}$ II regions that have a nearly uniform metallicity, $\langle 12+\log (\mathrm{O} / \mathrm{H})\rangle=8.37$ (for the main body), higher than expected given the stellar luminosity if AGC 226067 were a dwarf galaxy (Beccari et al. 2017a). The H II region velocities of the main and secondary bodies agree with the H I clouds and the overall system displays a weak velocity gradient that spans both bodies (Adams et al. 2015; Beccari et al. 2017a). Perhaps most intriguingly, there is no old stellar population associated with AGC 226067 because the entire diffuse light budget can be accounted for by the expected faintend luminosity function of the visible $\sim 7-50 \mathrm{Myr}$ stellar population. Given the metallicity and lack of old stellar population, AGC 226067 is a strong tidal dwarf candidate, and its extreme isolation makes it a unique example of this class of objects.

In understanding the origin of AGC 226067 we must also keep in mind that there seem to be other stellar systems of similar appearance in the NGVS data set (Section 4), and perhaps a yet larger population of older, undetectable systems, as well as Virgo H I cloud complexes that have no stellar counterpart at all (e.g., Kent 2010; Taylor et al. 2012)—-there is plausibly a continuum of objects that are $\mathrm{H} \mathrm{I-rich} \mathrm{and} \mathrm{star-poor}$ (see also the "Almost Dark" objects; Cannon et al. 2015). Recent theoretical effort has sought to understand the physics (e.g., Burkhart \& Loeb 2016) and possible origins of these clouds (e.g., Taylor et al. 2016); here we suggest that AGC 226067 is a stripped remnant of the numerous interactions in the Virgo cluster environment, most plausibly associated with the M86 subgroup.

\subsection{A Remnant of M86 Subgroup Interactions}

The removal of gas from cluster galaxies due to the hot intracluster medium (ICM) via ram pressure stripping (Gunn \& Gott 1972) is one plausible mechanism that could explain the origin of AGC 226067. Recent simulations of ram pressure stripping have shown that small amounts of star formation in the stripped tail of ablated gas can be seen up to hundreds of kiloparsecs from the galaxy of origin (Kapferer et al. 2009; Tonnesen \& Bryan 2012). This star formation is not from stripped molecular clouds of gas from the parent galaxy, but is low density stripped gas that cools and condenses in the turbulent wake of the stripping, a process that takes $\sim 300-750$ Myr at the pressure of the Virgo ICM. Individual star-forming knots contain up to $M_{\text {stars }} \sim 10^{5-6} M_{\odot}$, similar to AGC 226067, and is not expected to contribute significantly to the total intracluster light budget (Kapferer et al. 2009; Tonnesen \& Bryan 2012). There are examples of intracluster star formation (or implied star formation from $\mathrm{H} \alpha$ emission) due to ram pressure stripping in nearby galaxy clusters (e.g., Kenney \& Koopmann 1999; Cortese et al. 2007; Yoshida et al. 2008; Sun et al. 2010; Yagi et al. 2010; Kenney et al. 2014, among others), but to our knowledge none have been uncovered at such large projected distances $(D \sim 350 \mathrm{kpc})$ from their point of origin as AGC 226067; systems such as this and those uncovered in this work can provide an important test of ram pressure models.

In Figure 4, we show AGC 226067 in relation to the M86 subgroup of the Virgo cluster, a major component of the "Low Velocity Cloud" of Virgo galaxies with similar low heliocentric velocities as AGC 226067. AGC 226067 is $\sim 350 \mathrm{kpc}$ in projection from M86. Overplotted are the Chandra surface brightness contours (Randall et al. 2008) and $\mathrm{H} \alpha$ contours (Kenney et al. 2008) surrounding both M86 and NGC 4438, which show evidence for a previous interaction with each other 
(e.g., Kenney et al. 2008), and are likely undergoing a ram pressure stripping event as they fall into the larger Virgo cluster together (Randall et al. 2008; Ehlert et al. 2013, among others). The stream of X-ray emitting gas and $\mathrm{H} \alpha$ emission point in the general direction of AGC 226067, but the extent of the available data sets is not large enough to probe out to the necessary distances, as marked in Figure 4. Given the suggestive stream pointing in the direction of AGC 226067, this interpretation of its origin is our preference, though future $\mathrm{X}$-ray and $\mathrm{H} \alpha$ mapping will be necessary to confirm this scenario.

Beccari et al. (2017a) suggested that the interacting galaxy pair $\operatorname{NGC} 4299\left(v_{\odot} \approx+230 \mathrm{~km} \mathrm{~s}^{-1}\right)$ and $\operatorname{NGC~} 4294\left(v_{\odot} \approx\right.$ $+350 \mathrm{~km} \mathrm{~s}^{-1}$ ) were a plausible origin for AGC 226067 if it were a tidal remnant. We find this scenario to be less likely than that of an M86 subgroup origin because of the greater projected distance to NGC $4299+$ NGC 4294 ( 600 kpc), the larger velocity discrepancy between this pair and AGC 226067, and because the $\mathrm{H}$ I tidal tail morphology of that pair is pointed to the southwest, nearly in the opposite direction of AGC 226067 (Chung et al. 2009), which is almost due north.

\section{Conclusions}

We have presented HST imaging of the UCHVC AGC 226067, an enigmatic gas-rich stellar system in the Virgo cluster. The HST data reveal an exclusively young stellar population of $\sim 7-50 \mathrm{Myr}$ and $\mathrm{a}[\mathrm{Fe} / \mathrm{H}] \sim-0.3$, in contrast to normal dwarf galaxy systems, which always show some old stellar population $(\geq 5 \mathrm{Gyr})$ upon close inspection. Based on these HST results and other results in the literature on this object (see the discussion in Section 5), there is circumstantial evidence that AGC 226067 is a distant star-forming remnant of the ram pressure stripping event in the M86 subgroup, as recent simulations have predicted (Kapferer et al. 2009; Tonnesen \& Bryan 2012).

Our initial search for objects with similar optical/UV properties in the NGVS turned up two objects in $20 \mathrm{deg}^{2}$, but it is likely that we are only sensitive to the youngest and most luminous examples of this emerging class of stellar objects. Follow-up HI, X-ray, and $\mathrm{H} \alpha$ observations of AGC 226067 and its potential brethren will help elucidate their physical nature.

D.J.S. is supported by NSF grants AST-1412504 and AST1517649. K.S. acknowledges support from the Natural Science and Engineering Research Council of Canada. E.A.K.A. is supported by TOP1EW.14.105, which is financed by the Netherlands Organisation for Scientific Research (NWO). E.T. and P.G. acknowledge the NSF grants AST-1010038 and AST-1412504. J.S. acknowledges support from the Packard Foundation. We thank the Aspen Center for Physics (NSF Grant \#1066293) for their hospitality during the writing of this paper and the 2016 WoA committee for its support. Support for program \#13735 was provided by NASA through a grant from the Space Telescope Science Institute, which is operated by the Association of Universities for Research in Astronomy, Inc., under NASA contract NAS 5-26555.

\section{References}

Adams, E. A. K., Faerman, Y., Janesh, W. F., et al. 2015, A\&A, 573, L3 Adams, E. A. K., Giovanelli, R., \& Haynes, M. P. 2013, ApJ, 768, 77

Adams, E. A. K., Oosterloo, T. A., Cannon, J. M., Giovanelli, R., \& Haynes, M. P. 2016, A\&A, 596, 117

Baumgardt, H. 2017, MNRAS, 464, 2174

Beccari, G., Bellazzini, M., Battaglia, G., et al. 2016, A\&A, 591, A56

Beccari, G., Bellazzini, M., Magrini, L., et al. 2017a, MNRAS, 465, 2189

Beccari, G., Bellazzini, M., Magrini, L., et al. 2017b, MNRAS, 468, 4254

Bellazzini, M., Beccari, G., Battaglia, G., et al. 2015a, A\&A, 575, A126

Bellazzini, M., Magrini, L., Mucciarelli, A., et al. 2015b, ApJL, 800, L15

Bigiel, F., Leroy, A., Walter, F., et al. 2008, AJ, 136, 2846

Bigiel, F., Leroy, A., Walter, F., et al. 2010, AJ, 140, 1194

Boselli, A., Voyer, E., Boissier, S., et al. 2014, A\&A, 570, A69

Bressan, A., Marigo, P., Girardi, L., et al. 2012, MNRAS, 427, 127

Bruzual, G., \& Charlot, S. 2003, MNRAS, 344, 1000

Burkhart, B., \& Loeb, A. 2016, ApJL, 824, L7

Cannon, J. M., Martinkus, C. P., Leisman, L., et al. 2015, AJ, 149, 72

Chung, A., van Gorkom, J. H., Kenney, J. D. P., Crowl, H., \& Vollmer, B. 2009, AJ, 138, 1741

Cortese, L., Marcillac, D., Richard, J., et al. 2007, MNRAS, 376, 157

Dohm-Palmer, R. C., \& Skillman, E. D. 2002, AJ, 123, 1433

Dolphin, A. E. 2000, PASP, 112, 1383

Duc, P. A. 2012, AASP, 28, 305

Ehlert, S., Werner, N., Simionescu, A., et al. 2013, MNRAS, 430, 2401

Ferrarese, L., Côté, P., Cuillandre, J.-C., et al. 2012, ApJS, 200, 4

Ford, H. C., Bartko, F., Bely, P. Y., et al. 1998, Proc. SPIE, 3356, 234

Giovanelli, R., \& Haynes, M. P. 2016, A\&ARv, 24, 1

Giovanelli, R., Haynes, M. P., Kent, B. R., \& Adams, E. A. K. 2010, ApJL, 708, L22

Graham, M. L., Sand, D. J., Bildfell, C. J., et al. 2012, ApJ, 753, 68

Grevesse, N., Asplund, M., \& Sauval, A. J. 2007, SSRv, 130, 105

Gunn, J. E., \& Gott, J. R., III 1972, ApJ, 176, 1

Gwyn, S. D. J. 2008, PASP, 120, 212

Haynes, M. P., Giovanelli, R., Martin, A. M., et al. 2011, AJ, 142, 170

Janesh, W., Rhode, K. L., Salzer, J. J., et al. 2015, ApJ, 811, 35

Kapferer, W., Sluka, C., Schindler, S., Ferrari, C., \& Ziegler, B. 2009, A\&A, 499,87

Kenney, J. D. P., Geha, M., Jáchym, P., et al. 2014, ApJ, 780, 119

Kenney, J. D. P., \& Koopmann, R. A. 1999, AJ, 117, 181

Kenney, J. D. P., Tal, T., Crowl, H. H., Feldmeier, J., \& Jacoby, G. H. 2008, ApJL, 687, L69

Kennicutt, R. C. 1998, ApJ, 498, 541

Kent, B. R. 2010, ApJ, 725, 2333

Kroupa, P. 2001, MNRAS, 322, 231

Martin, D. C., Fanson, J., Schiminovich, D., et al. 2005, ApJL, 619, L1

McQuinn, K. B. W., Cannon, J. M., Dolphin, A. E., et al. 2014, ApJ, 785,3

McQuinn, K. B. W., Skillman, E. D., Dalcanton, J. J., et al. 2011, ApJ, 740, 48

Randall, S., Nulsen, P., Forman, W. R., et al. 2008, ApJ, 688, 208

Sand, D. J., Crnojević, D., Bennet, P., et al. 2015, ApJ, 806, 95

Sand, D. J., Crnojević, D., Strader, J., et al. 2014, ApJL, 793, L7

Saul, D. R., Peek, J. E. G., Grcevich, J., et al. 2012, ApJ, 758, 44

Schlafly, E. F., \& Finkbeiner, D. P. 2011, ApJ, 737, 103

Sirianni, M., Jee, M. J., Benítez, N., et al. 2005, PASP, 117, 1049

Strader, J., Caldwell, N., \& Seth, A. C. 2011, AJ, 142, 8

Sun, M., Donahue, M., Roediger, E., et al. 2010, ApJ, 708, 946

Taylor, R., Davies, J. I., Auld, R., \& Minchin, R. F. 2012, MNRAS, 423, 787

Taylor, R., Davies, J. I., Jáchym, P., et al. 2016, MNRAS, 461, 3001

Thilker, D. A., Bianchi, L., Meurer, G., et al. 2007, ApJS, 173, 538

Tollerud, E. J., Geha, M. C., Grcevich, J., Putman, M. E., \& Stern, D. 2015, ApJL, 798, L21

Tonnesen, S., \& Bryan, G. L. 2012, MNRAS, 422, 1609

Williams, B. F., Ciardullo, R., Durrell, P. R., et al. 2007, ApJ, 656, 756

Williams, B. F., Lang, D., Dalcanton, J. J., et al. 2014, ApJS, 215, 9

Yagi, M., Yoshida, M., Komiyama, Y., et al. 2010, AJ, 140, 1814

Yoshida, M., Yagi, M., Komiyama, Y., et al. 2008, ApJ, 688, 918 